\title{
Tilted Bianchi Type I Barotropic Cosmological Model
}

\author{
Anita Bagora and Rakeshwar Purohi
}

\begin{abstract}
Tilted Bianchi type I cosmological model with barotropic fluid distribution and heat conduction is investigated. To get deterministic solution, we have assumed barotropic condition $(p=\gamma \rho)$, where $\mathbf{p}$ being isotropic pressure, $\rho$ the matter density with $0 \leq \gamma \leq 1$.Also, we assumed that the expansion in the model is only in two directions i.e. one of the component of Hubble parameter $\left(H_{1}=\frac{A_{4}}{A}\right)$ is zero. The physical and geometrical aspects of the model are also discussed.
\end{abstract}

Index Terms — Tilted cosmological model; bianchi type-I universe; barotropic fluid.

\section{INTRODUCTION}

Homogeneous and anisotropic cosmological models have been widely studied in classical general relativity in the search for a relativistic picture of the universe in its early stages because they can be explained a number of observed phenomena quite satisfactorily. So that in recent years, there has been a considerable interest in investigating spatially homogeneous and anisotropic cosmological models in which mater do not move orthogonally to the hyper surface of homogeneity. These types of models are called tilted cosmological models. The general dynamics of these cosmological models have been studied in details by King and Ellis [1] and they have shown that in such universe, the matter move with non-zero expansion, rotation and shear. Also, Ellis and King [2], Collins and Ellis [3], Ellis and Baldwin [4] have shown that we are likely to be living in a tilted universe and they have indicated that how we may detect it.

The Bianchi models can be used to analyze aspects of the physical universe which pertain to or which may be affected by anisotropy in the rate of expansion.Bianchi type I cosmological models have been studied by several authors in various context viz. Mazumdar [5], Aguirregabiria [6], Yavuz [7], Beesham [8]. Johri et al. [9] investigated the general Bianchi type I cosmological solution corresponding to spatially homogeneous and anisotropic models containing barotropic fluid.Cosmological models with heat flow have been studied by various author viz. Novello and Reboucas [10], Ray [11], Reboucas and Lima [12], Roy and Banerjee $[13,14]$.Bagora [15-16] obtained tilted Bianchi type I and III cosmological model for disordered radiation and stiff

Manuscript received June 6, 2012, revised June 18, 2012.

Anita Bagora is with Jaipur National University, Jaipur-302025, India (e-mail: anitabagora@gmail.com).

Rakeshwar Purohit is with M.L. Sukhadia University, Udaipur-313001, India (email: ramkrishnadr@gmail.com). fluid distribution. Motivated by these studies, in this paper we propose to find tilted Bianchi type I cosmological model for barotropic fluid.Also, we assumed that the expansion in the model is only in two directions i.e. one of the component of Hubble parameter is zero. The physical and geometrical aspects of the model are also discussed.

\section{THE FIELD EQUATIONS}

We consider the Bianchi type-I metric in the form

$$
\mathrm{ds}^{2}=-\mathrm{dt}^{2}+\mathrm{dx}^{2}+B^{2} \mathrm{dy}^{2}+C^{2} \mathrm{dz}^{2}
$$

where $\mathrm{B}$ and $\mathrm{C}$ are functions of ' $\mathrm{t}$ ' alone.

The energy-momentum tensor for perfect fluid distribution with heat conduction given by Ellis [17] is given by

$$
T_{i}^{j}=(\rho+p) v_{i} v^{j}+p g_{i}^{j}+q_{i} v^{j}+v_{i} q^{j}
$$

Together with

$$
\begin{aligned}
& \text { gijv }^{\mathrm{i}} v j=-1 \\
& q^{\mathrm{i}} q^{\mathrm{i}}>0 \\
& q i v i=0
\end{aligned}
$$

In the above, $p$ is the isotropic pressure, $\rho$ the matter density, qi the heat conduction vector orthogonal to vi. The fluid flow vector $v i$ has the components $(\sinh \lambda, 0,0, \cosh$ $\lambda$ ) satisfying (3), $\lambda$ being the tilt angle.

The Einstein's field equation

$$
R_{i}^{j}-\frac{1}{2} \operatorname{Rg}_{i}^{j}=-8 \pi T_{i}^{j}, \quad(c=G=1)
$$

The field equation for the line element (1) leads to

$$
\begin{gathered}
\frac{B_{44}}{B}+\frac{C_{44}}{C}+\frac{\mathrm{B}_{4} C_{4}}{\mathrm{BC}}=-8 \pi\left[(\rho+p) \sinh ^{2} \lambda+p+2 q_{1} \sinh \lambda\right] \\
\frac{C_{44}}{C}=-8 \pi p \\
\frac{B_{44}}{B}=-8 \pi p \\
\frac{B_{4} C_{4}}{B C}=-8 \pi\left[-(\rho+p) \cosh ^{2} \lambda+p-2 q_{1} \sinh \lambda\right] \\
(\rho+p) \sinh \lambda \cosh \lambda+q_{1} \cosh \lambda+q_{1} \frac{\sinh ^{2} \lambda}{\cosh \lambda}=0
\end{gathered}
$$

where the suffix ' 4 ' stands for ordinary differentiation with respect to the cosmic time ' $t$ ' alone. 


\section{Solution OF THE Field EQuations}

Equations from (6)-(10) are five equations in six unknown B, C $\rho, \mathrm{p}, q_{1}$ and $\lambda$.For the complete determination of these quantities, we assume that the model is filled with barotropic perfect fluid which leads to

$$
p=\gamma \rho
$$

where $0 \leq \gamma \leq 1$.

Equations (6) and (9) with (11) lead to

$$
\frac{B_{44}}{B}+\frac{C_{44}}{C}+\frac{2 \mathrm{~B}_{4} \mathrm{C}_{4}}{\mathrm{BC}}=8 \pi\left[\left(\frac{p}{\gamma}-p\right)\right]
$$

Using (8) in (12), we have

$$
\frac{B_{44}}{B}+\frac{C_{44}}{C}+\frac{2 \mathrm{~B}_{4} \mathrm{C}_{4}}{\mathrm{BC}}=-\frac{B_{44}}{B}\left(\frac{1}{\gamma}-1\right)
$$

Equations (7) and (8) lead to

$$
\frac{B_{44}}{B}-\frac{C_{44}}{C}=0
$$

Let us assume that

$$
B C=\mu \quad \frac{\mathrm{B}}{C}=\nu
$$

With the help of (15), equation (14) leads to

$$
\frac{\nu_{4}}{\nu}=\frac{a}{\mu}
$$

where ' $a$ ' is constant of integration.

Using (15) and (16) in (13), we have

$$
2 \mu_{44}+\alpha \frac{\mu_{4}^{2}}{\mu}=\frac{\alpha a^{2}}{\mu}
$$

where $\quad \alpha=\frac{\gamma-1}{\gamma+1}$

Equation (17) gives

$$
\mu_{4}^{2}=a^{2}+\frac{b}{\mu^{\alpha}}
$$

where ' $\mathrm{b}$ ' is constant of integration and $\mu_{4}=f(\mu)$ Equation (18) leads to

$$
\log \nu=\int \frac{\mathrm{ad} \mu}{\mu \sqrt{a^{2} \mu^{\alpha}+b}}
$$

Hence the metric (1) reduces to the form

$$
\mathrm{ds}^{2}=-\frac{\mathrm{d} \mu^{2}}{\mathrm{f}^{2}}+d x^{2}+\mu \nu d y^{2}+\frac{\mu}{\nu} d z^{2}
$$

By introducing the following transformations

$$
\mu=T, x=X, y=Y, z=Z \text {. }
$$

The metric (20) reduces to the form

$$
\mathrm{ds}^{2}=\frac{-T^{\alpha} d T^{2}}{a^{2} T^{\alpha}+b}+d X^{2}+\mathrm{T} \nu \mathrm{dY}^{2}+\frac{\mathrm{T}}{\nu} d Z^{2}
$$

where $\nu$ is determined by (19) when $\mu=T$.

\section{SOME Physical AND GeOMETRICAL FeAtures}

The matter density $\rho$ and isotropic pressure $p$ for the model (21) are given by

$$
\begin{aligned}
& 8 \pi p=\frac{\gamma b}{2(1+\gamma) T^{3 \gamma+1 / \gamma+1}} \\
& 8 \pi \rho=\frac{b}{2 \gamma(1+\gamma) T^{3 \gamma+1 / \gamma+1}}
\end{aligned}
$$

The tilt angle $\lambda$ is given by

$$
\begin{aligned}
& \cosh \lambda=\sqrt{\frac{(1+3 \gamma)}{2(1+\gamma)}} \\
& \sinh \lambda=\sqrt{\frac{(\gamma-1)}{2(1+\gamma)}}
\end{aligned}
$$

The scalar of expansion $\theta$ calculated for the flow vector vi for the model (21) is given by

$$
\theta=\frac{1}{\mathrm{~T}} \sqrt{\frac{(1+3 \gamma)\left(a^{2} \mathrm{~T}^{\alpha}+\mathrm{b}\right)}{2(1+\gamma) T^{\alpha}}}
$$

The flow vectors vi and heat conduction vectors $q_{i}$ for the model (21) are given by

$$
\begin{gathered}
\nu^{1}=\sqrt{\frac{(\gamma-1)}{2(1+\gamma)}} \\
v^{4}=\sqrt{\frac{(1+3 \gamma)}{2(1+\gamma)}} \\
q_{1}=\frac{-\gamma(1+3 \gamma) b}{16 \pi(1+\gamma)^{2} T^{3 \gamma+1 / \gamma+1}} \sqrt{\frac{\gamma-1}{2(\gamma+1)}} \\
q_{4}=\frac{\gamma(\gamma-1) b}{16 \pi(1+\gamma)^{2} T^{3 \gamma+1 / \gamma+1}} \sqrt{\frac{3 \gamma+1}{2(\gamma+1)}}
\end{gathered}
$$

The non-vanishing components of shear tensor ( $\sigma \mathrm{ij})$ are

$$
\begin{gathered}
\sigma_{11}=\frac{-(3 \gamma+1)}{12(\gamma+1) \mathrm{T}} \sqrt{\frac{(1+3 \gamma)\left(a^{2} T^{\alpha}+b\right)}{2(1+\gamma) T^{\alpha}}} \\
\sigma_{14}=\frac{(3 \gamma+1)}{12(\gamma+1) \mathrm{T}} \sqrt{\frac{(\gamma-1)\left(a^{2} T^{\alpha}+b\right)}{2(1+\gamma) T^{\alpha}}} \\
\sigma_{11} \nu^{1}+\sigma_{14} \nu^{4}==0
\end{gathered}
$$

The physical significance of conditions (33) is explained by Ellis [18]: The shear tensor $\left(\sigma_{i j}\right)$ determines the distortion arising in the fluid flow, leaving the volume invariant. The direction of principal axis is unchanged by the distortion, but all other directions are changed. Thus, we have

Which leads to $\sigma_{i j} \nu^{j}=0$

$$
\sigma_{11} \nu^{1}+\sigma_{14} \nu^{4}=0 \quad\left(\because \nu_{1} \neq 0, \nu_{4} \neq 0\right)
$$

Shear $(\sigma)$ is given by 


$$
\sigma^{2}=\frac{1}{2} \sigma_{i j} \sigma^{i j}
$$

Thus $\sigma^{2} \geq 0$ and $\sigma=0 \Leftrightarrow \sigma_{i j}=0$

\section{CONCLUSION}

The reality conditions $\rho+p>0, \rho+3 p>0$ given by Ellis [19] lead to $b>0, \gamma+1>0$ or $b<.0 \gamma+1<0$ ie these conditions hold good when $\gamma=0$ i.e. $p=0$ The matter density $\rho \rightarrow \infty$ when $T \rightarrow 0$ and $\rho \rightarrow 0$ when $T \rightarrow \infty$ therefore $\rho$ is the decreasing function of time. The model starts with a big-bang at $T=0$ and the expansion in the model decreases as time increases. Also, the velocity components $\nu^{1}$ and $\nu^{4}$ are constant i.e. flow is uniform and tilt angle is independent of time. The condition $\sigma_{i j} \nu^{j}=0$ is satisfied as $\sigma_{11} \nu^{1}+\sigma_{14} \nu^{4}=0$. The model has cigar type singularity at $T=0$ when $\alpha>0$ and it has point type singularity at $T=0$ when $\alpha<0$ (MacCallum, [20]). Also, the declaration parameter $q=-1+\frac{\left[\alpha a^{2} T^{2}+b \alpha+2 b\right]}{3 T \sqrt{T^{\alpha+2}\left(a^{2} T^{2}+b\right)}}$

Implies an accelerating model of the universe. Recent observations of type Ia supernovae [21], [22] reveal that the present universe is in accelerating phase and deceleration parameter lies somewhere in the range $1<q \leq 0$. It also follows that our model of the universe consistent with recent observations. The deceleration parameter $q$ approaches the value (1) as in the case of de-Sitter universe.

\section{REFERENCES}

[1] A. R. King and G. F. R. Ellis, Comm. Math. Phys, vol. 31, pp. 209242, 1973.

[2] G. F. R. Ellis and A. R. King, Comm. Math. Phys, vol. 38, no. 2, pp. 119-156, 1974.

[3] C. B. Collins and G. F. R. Ellis, Phys. Rep, vol. 56, pp. 65-105, 1979.

[4] G. F. R. Ellis and J.E. Baldwin, Mon. Not. Roy. Astron. Soc, vol. 206, pp. 377-381, 1984.

[5] A. Mazumdar, Gen. Relativ. Gravit, vol. 26, no. 3, pp. 307-310, 1994.

[6] J. M. Aguirregabiria and L. P. Chimento, Class. Quantum Gravit, vol. 13, no. 12, pp. 3197-3209, 1996.

[7] I. Yavuz and I. Tarhan, Astrophys. Space Sc, vol. 240, no. 1, pp. 4554, 1996.

[8] A. Beesham, Gen. Relativ. Gravit, vol. 26, no. 2, pp. 159-165, 1994,

[9] V. B. Johri, G. K. Goswami, and I. J. Singh, Ind. J. Pure and App. Math, vol. 12, no. 6, pp.786-797, 1981.

[10] M. Novello and M. J. Reboucas, Astrophys. J, pp. 719-724, 1978.

[11] D. J. Ray, Math. Phys, vol. 2797-2798, 1980.

[12] M. J. Reboucas and J. A.S. Lima, J. Math. Phys, vol. 23, pp. 1547. 1982 ,

[13] S. R. Roy and S. K. Banerjee, Astrophys Space Sci, vol. 150, no. 2, 213-222, 1988.

[14] S. R. Roy and S. K. Banerjee, Gen. Relativ. Gravit, vol. 28, no. 1, 2733, 1996.

[15] A. Bagora. EJTP, vol. 4, no. 14, pp. 373-382, 2010.

[16] A. Bagora, Astrophys. Space Sci, vol. 319, pp. 155-159, 2009.

[17] G. F. R. Ellis, General Relativity and Cosmology; ed. R. K. Sachs; Academic Press; New York, pp.116, 1971.

[18] G. F. R. Ellis, General Relativity and Cosmology; ed. R.K. Sachs; Academic Press; New York, pp. 113, 1971.

[19] G. F. R. Ellis, General Relativity and Cosmology; ed. R.K. Sachs; Academic Press; New York, pp. 117, 1971.

[20] M. A. H. M. Callum, Comm. Maths. Phys, vol. 20, no. 57, 1971.

[21] S. Perlmutter et al., Astrophysic. J, vol. 483, pp. 565.

[22] S. Perlmutter et al., Nature, vol. 391, no. 51, 1998.

[23] S. Perlmutter et al., Astrophysics J, vol. 517, no. 565, 1999.

[24] A. G. Reiss et al., Astron. $J$, vol. 116, pp. 1009, 1998.

[25] A. G. Reiss et al., Astron. J, vol. 607, pp. 665, 2004. 